Bull. Austral. Math. Soc.

$26 \mathrm{~A} 18,58 \mathrm{~F} 22,54 \mathrm{H} 20$

VOL. 49 (1994) [469-481]

\title{
REPELLING INVARIANT CURVES IN PLANAR DISCRETE DYNAMICAL SYSTEMS
}

\author{
Francisco Esquembre
}

\begin{abstract}
Constructive, simple proofs for the existence, regularity, continuous dependence and dynamical properties of a repelling invariant curve for a discrete dynamical system of the plane with an attracting fixed point with real eigenvalues are given. These proofs can be used to generate a numerical algorithm to find these curves and to compute explicitly the dependence of the curve with respect to the system.
\end{abstract}

\section{INTRODUCTION}

A common and useful way to understand the behaviour of the dynamical system generated by the iteration of a map of $\mathbb{R}^{n}(n=1$ or 2$)$ into itself is to find simple structures in the phase space of the system and to describe the dynamics in terms of the effect caused by the presence of these structures. Typical structures are fixed and periodic points and, in dimension two, invariant curves and circles. For these structures to be of dynamical relevance, a kind of attracting or repelling behaviour is required, and, usually, a certain regularity is also of interest. Most commonly, changes in parameters governing the system may cause these curves to change form, behaviour or, simply, appear or disappear following different kinds of bifurcations.

In this paper we shall study repelling invariant curves coming through fixed (or periodic) attracting points of a two dimensional map such that the eigenvalues of the differential of the map at the fixed point are both real and their absolute values are different and smaller than one. Aronson, Chory, Hall and McGehee made clear in [1] that the precise determination of the behaviour of orbits near the fixed point (apart from the obvious convergence to it) is of importance for the description of the changes in smoothness and even total break of invariant circles born in a Hopf bifurcation. The results we give in this paper can be used for this determination (see [2]).

As a new aspect, we consider the case where the iterates arc composed of possibly different maps $T_{n}$, while in the classical theory, all the $T_{n}$ are equal. In this particular case, many (but not all) of our results can be found in [4]. However, our proofs use elementary estimates, while in [4] knowledge of manifolds and vector bundles is presupposed. Although the existence has been proved elsewhere (see [3] for the unstable curve and [5] for the stable one), it has been done in a different context.

Received 4 August 1993

Copyright Clearance Centre, Inc. Serial-fee code: 0004-9729/94 \$A2.00+0.00. 
To our knowledge, the precise determination of the dependence with respect to the map describing the dynamics that we give in Proposition 4 is new. Also, our proof of the existence of the unstable curve is constructive and can be used in numerical studies.

The main results are obtained for the case in which the fixed point is the origin and the principal directions of the linearised model the $O X$ and $O Y$ axis. Theorem 6 summarises all the results for the general case.

All through this paper $n \geqslant 0$ means that $n$ is a non negative positive integer. We shall consider families of maps $\left\{T_{n}: \mathbb{R}^{2} \rightarrow \mathbb{R}^{2} \mid n \geqslant 0\right\}$ of the plane and denote $T^{n}=T_{n} \circ T_{n-1} \circ \ldots \circ T_{0}$, for all $n \geqslant 0$. Given any $\left(x_{0}, y_{0}\right) \in \mathbb{R}^{2}$, we shall denote by $\left(x_{n+1}, y_{n+1}\right)=T^{n}\left(x_{0}, y_{0}\right)$, for all $n \geqslant 0$. The set $\left\{\left(x_{n}, y_{n}\right): n \in \mathbb{N}\right\}$ (or simply $\left.\left(x_{n}, y_{n}\right)_{n}\right)$ will be called the orbit under the family $\left\{T_{n}: n \geqslant 0\right\}$ (or simply $\left(T_{n}\right)_{n}$ ) with initial condition $\left(x_{0}, y_{0}\right)$.

\section{EXISTENCE AND PROPERTIES OF A REPELLING INVARIANT CURVE}

We shall need the following topological lemma.

LEMMA 1. Let $\left\{T_{n}: \mathbb{R}^{2} \rightarrow \mathbb{R}^{2} \mid n \geqslant 0\right\}$ be a family of continuous maps of the plane. Let $F$ and $K$ be two subsets of $\mathbb{R}^{2}, F$ closed and $K$ compact, satisfying

$$
\begin{gathered}
T_{n}\left(\mathbb{R}^{2}-F\right) \subseteq \mathbb{R}^{2}-F, \text { for all } n \geqslant 0 \\
F \cap T^{n}(K) \neq \emptyset, \text { for all } n \geqslant 0
\end{gathered}
$$

Then, there exists $p_{0} \in K$ such that $T^{n}\left(p_{0}\right) \in F$, for all $n \geqslant 0$.

Proof: Consider the family of closed subsets of $K$ given by $G_{n}=\left(T^{n}\right)^{-1}\left(F \cap T^{n}(K)\right) \cap K$. Then, $G_{n} \neq \emptyset, G_{n}$ is compact and $G_{n+1} \subseteq G_{n}$, since if $x \in G_{n+1}$, then $T^{n+1}(x)=T_{n+1}\left(T^{n}(x)\right) \in F \cap T^{n+1}(K)$; hence $T^{n}(x) \in F \cap T^{n}(K)$ and $x \in G_{n}$. Take now $p_{0} \in \bigcap_{n \geqslant 0} G_{n}(\neq 0)$, then $T^{n}\left(p_{0}\right) \in F$, for all $n \geqslant 0$.

PROPOSITION 2. Let $\left\{T_{n}: \mathbb{R}^{2} \rightarrow \mathbb{R}^{2} \mid n \geqslant 0\right\}$ be a family of $C^{1}$ maps that can be written in the form $T_{n}(x, y)=\left(a x+f_{n}(x, y), b y+g_{n}(x, y)\right)$, and such that

(2.1) $f_{n}(0,0)=g_{n}(0,0)=0$, for all $n \geqslant 0$ (the origin is a fixed point of $T_{n}$ ),

(2.2) given any fixed $n$ and $x_{0}$, the mapping $\alpha: \mathbb{R} \rightarrow \mathbb{R}, \alpha(y)=y_{n}$ (where $\left.\left(x_{n}, y_{n}\right)=T^{n-1}\left(x_{0}, y\right)\right)$, has at least one zero,

(2.3) there exists $0<\varepsilon \leqslant(|b|-|a|) / 2$ such that $\left\|D f_{n}(x, y)\right\| \leqslant \varepsilon$ and $\left\|D g_{n}(x, y)\right\| \leqslant \varepsilon$, for every $(x, y) \in \mathbb{R}^{2}$ and every $n \geqslant 0$.

Then, if we denote by $F$ the closed set $\left\{(x, y) \in \mathbb{R}^{2}|| y|\leqslant| x \mid\right\}$, the following statements hold,

(2.a) if $\left\{\left(x_{n}, y_{n}\right)\right\}_{n \geqslant 0} \subseteq F$, then $\left|y_{n}\right| \leqslant\left|x_{n}\right| \leqslant(|a|+\varepsilon)^{n}\left|x_{0}\right|$, for all $n \geqslant 0$, 
(2.b) if, on the contrary, there exists $n_{0}$ such that $\left(x_{n_{0}}, y_{n_{0}}\right)$ does not belong to $F$, then $\left|y_{n}\right| \geqslant k(|b|-\varepsilon)^{n}$, for every $n \geqslant n_{0}$, with $k$ a positive constant,

(2.c) for every $x_{0} \in \mathbb{R}$, there exists $y_{0},\left|y_{0}\right| \leqslant\left|x_{0}\right|$, such that $\left\{\left(x_{n+1}, y_{n+1}\right)=\right.$ $\left.T^{n}\left(x_{0}, y_{0}\right)\right\}_{n \geqslant 0} \subseteq F$.

Notice that (2.3) implies $|a|<|b|$.

Proof: We note that for every $(x, y) \in \mathbb{R}^{2}$ the following inequalities hold,

$$
\begin{aligned}
\left|b y+g_{n}(x, y)\right| & \geqslant|b||y|-\left|g_{n}(x, y)-g_{n}(0,0)\right| \\
& =|b||y|-\left|D g_{n}\left(\xi_{1}, \xi_{2}\right)(x, y)\right| \\
& \geqslant|b||y|-\left\|D g_{n}\left(\xi_{1}, \xi_{2}\right)\right\|\|(x, y)\| \\
& \geqslant|b||y|-\varepsilon\|(x, y)\|,
\end{aligned}
$$

for some $\left\|\left(\xi_{1}, \xi_{2}\right)\right\| \leqslant\|(x, y)\|$, and

$$
\left|a x+f_{n}(x, y)\right| \leqslant|a||x|+\varepsilon\|(x, y)\| .
$$

$F$ is a closed set such that $T_{n}\left(\mathbb{R}^{2}-F\right) \subseteq \mathbb{R}^{2}-F$ since, if $(x, y) \notin F$, then $|y|>|x|$ and, since $\left|b y+g_{n}(x, y)\right| \geqslant(|b|-\varepsilon)\|(x, y)\|$ and $\left|a x+f_{n}(x, y)\right| \leqslant(|a|+\varepsilon)\|(x, y)\|$, $|a|+\varepsilon \leqslant|b|-\varepsilon$ yields $T_{n}(x, y) \notin F$.

Given $x_{0}$, we define $K\left(x_{0}\right)=\left\{\left(x_{0}, y\right) \in \mathbb{R}^{2}|| y|\leqslant| x_{0} \mid\right\} . K_{0}$ is a compact set satisfying $F \cap T^{n}\left(K\left(x_{0}\right)\right) \neq \emptyset$, for all $n \geqslant 0$, since for every $n$ and $x_{0},(2.2)$ gives the existence of $\bar{y}_{0} \in \mathbb{R}$ such that $T^{n}\left(x_{0}, \bar{y}_{0}\right)=\left(x_{n+1}, 0\right) \in F$. Moreover, $\left|\bar{y}_{0}\right| \leqslant\left|x_{0}\right|$ or, equivalently, $\left(x_{0}, \bar{y}_{0}\right) \in K\left(x_{0}\right)$, since $\left|\bar{y}_{0}\right|>\left|x_{0}\right|$ means $\left(x_{0}, \bar{y}_{0}\right) \notin F$ and then $T^{n}\left(x_{0}, y_{0}\right) \notin F$.

Using Lemma 1 with $\left(T_{n}\right)_{n}, F$ and $K\left(x_{0}\right)$ we obtain the existence of $p_{0}=$ $\left(x_{0}, y_{0}\right) \in K\left(x_{0}\right)$ such that $T^{n}\left(x_{0}, y_{0}\right) \in F$, for all $n \geqslant 0$. This gives (2.c).

To prove (2.a) we only need to notice that $\left(x_{n}, y_{n}\right) \in F$, for all $n \geqslant 0$, implies $\left\|\left(x_{n}, y_{n}\right)\right\|=\left|x_{n}\right|$ and, by (2.ii), $\left|x_{n+1}\right|=\left|a x_{n}+f_{n}\left(x_{n}, y_{n}\right)\right| \leqslant(|a|+\varepsilon)\left|x_{n}\right|$. By iterating this argument, $\left|x_{n}\right| \leqslant(|a|+\varepsilon)^{n}\left|x_{0}\right|$.

Now, if $\left(x_{n_{0}}, y_{n_{0}}\right) \notin F$, then $\left(x_{n}, y_{n}\right) \notin F$ for every $n \geqslant n_{0}$ and $\left\|\left(x_{n}, y_{n}\right)\right\|=\left|y_{n}\right|$ for every $n \geqslant n_{0}$. Hence, by $(2 . i)\left|y_{n+1}\right| \geqslant(|b|-\varepsilon)\left|y_{n}\right|$ and, by iterating this up to $n=n_{0}$,

$$
\left|y_{n}\right| \geqslant(|b|-\varepsilon)^{n-n_{0}}\left|y_{n_{0}}\right|=(|b|-\varepsilon)^{n} \frac{\left|y_{n_{0}}\right|}{(|b|-\varepsilon)^{n_{0}}}
$$

Taking $k=\left(\left|y_{n_{0}}\right|\right) /\left((|b|-\varepsilon)^{n_{0}}\right)$, we obtain (2.b).

We denote by $h_{u}$ the map defined from $\mathbb{R}$ into $\mathbb{R}$ by $h_{u}\left(x_{0}\right)=y_{0}$, where $y_{0}$ is a number such that $T^{n}\left(x_{0}, y_{0}\right) \in F$, for every $n \geqslant 0$. This map exists thanks to (2.c) 
and verifies that the orbits under the family $\left(T_{n}\right)_{n}$ of points of its graph remain in the cone $F$.

Since the linearised behaviour of any orbit would be analogous to $\left(a^{n} x_{0}, b^{n} y_{0}\right)$, and since $|a|<|b|$, this property is far from being trivial. The next result shows that, in fact, this property yields the uniqueness of $h_{u}$, and therefore the invariance of its graph under the action of the family $\left(T_{n}\right)_{n}$ and the global Lipschitz condition (with constant smaller than or equal to one) and thus continuity. Moreover, it is also proved that the curve is repelling in the sense of (3.b) below.

PROPOSITION 3. Let $\left(T_{n}\right)_{n \geqslant 0}$ be a family as in Proposition 2 and let $\left(x_{n}^{1}, y_{n}^{1}\right)_{n}$ and $\left(x_{n}^{2}, y_{n}^{2}\right)_{n}$ be two orbits under the family contained in $F$. Then,

$$
\left|y_{n}^{2}-y_{n}^{1}\right| \leqslant\left|x_{n}^{2}-x_{n}^{1}\right| \leqslant(|a|+\varepsilon)^{n}\left|x_{0}^{2}-x_{0}^{1}\right|, \text { for all } n \geqslant 0 .
$$

In particular, this yields the uniqueness of $h_{u}$. Let $\left(x_{n}, y_{n}\right)_{n}$ be an orbit under the family such that $y_{0} \neq h_{u}\left(x_{0}\right)$, then

$$
\left|y_{n}-h_{n}\left(x_{n}\right)\right| \geqslant(|b|-2 \varepsilon)^{n}\left|y_{0}-h_{u}\left(x_{0}\right)\right|>0, \text { for all } n \geqslant 0
$$

PROOF: Given $\left(T_{n}\right)_{n}$ and $\left(x_{n}^{1}, y_{n}^{1}\right)_{n}$, we consider the family of maps $\left(\triangle T_{n}\right)_{n}$, derived from the given one and defined for $n \geqslant 0$ by the formula

$$
\begin{aligned}
\Delta T_{n}(u, v)=\left(a u+\Delta f_{n}(u, v), b v+\Delta g_{n}(u, v)\right) & \\
=\left(a u+f_{n}\left(u+x_{n}^{1}, v+y_{n}^{1}\right)-f_{n}\left(x_{n}^{1}, y_{n}^{1}\right), b v+g_{n}\left(u+x_{n}^{1}, v+y_{n}^{1}\right)\right. & \left.-g_{n}\left(x_{n}^{1}, y_{n}^{1}\right)\right) .
\end{aligned}
$$

$\left(\triangle T_{n}\right)_{n}$ is of class $C^{1}$ and shares some of the properties if $\left(T_{n}\right)_{n}$. More precisely,

(3.i) $\triangle T_{n}\left(\mathbb{R}^{2}-F\right) \subseteq \mathbb{R}^{2}-F$

(3.ii) if $\left(u_{n_{0}}, v_{n_{0}}\right) \notin F$ then $\left|v_{n}\right| \geqslant k(|b|-\varepsilon)^{n}$, for all $n \geqslant n_{0}$.

This is true since $\Delta f_{n}(0,0)=\Delta g_{n}(0,0)=0,\left\|D\left(\Delta f_{n}\right)(u, v)\right\|=\| D f_{n}\left(u+x_{n}^{1}\right.$, $\left.v+y_{n}^{1}\right) \| \leqslant \varepsilon$, and $\left\|D\left(\triangle g_{n}\right)(u, v)\right\| \leqslant \varepsilon$. Hence, if $\left(u_{n}, v_{n}\right) \notin F$,

$$
\begin{aligned}
& \left|u_{n+1}\right|=\left|a u_{n}+\Delta f_{n}\left(u_{n}, v_{n}\right)\right| \leqslant|a|\left|u_{n}\right|+\varepsilon\left\|\left(u_{n}, v_{n}\right)\right\|=(|a|+\varepsilon)\left|v_{n}\right| \\
& \left|v_{n+1}\right|=\left|b v_{n}+\Delta g_{n}\left(u_{n}, v_{n}\right)\right| \geqslant|b|\left|v_{n}\right|-\varepsilon\left\|\left(u_{n}, v_{n}\right)\right\|=(|b|-\varepsilon)\left|v_{n}\right|
\end{aligned}
$$

and then, $\left(u_{n+1}, v_{n+1}\right) \notin F$. From here, we obtain (3.ii) as in the proof of Proposition 2.

Consider now the orbit with initial condition $\left(u_{0}, v_{0}\right)=\left(x_{0}^{2}-x_{0}^{1}, y_{0}^{2}-y_{0}^{1}\right)$. It is easy to see that then $\Delta T_{n}\left(x_{n}^{2}-x_{n}^{1}, y_{n}^{2}-y_{n}^{1}\right)=\left(x_{n+1}^{2}-x_{n+1}^{1}, y_{n+1}^{2}-y_{n+1}^{1}\right)$ 
and, hence, $\left(u_{n}, v_{n}\right)=\left(x_{n}^{2}-x_{n}^{1}, y_{n}^{2}-y_{n}^{1}\right)$, for all $n \geqslant 0$. Thus, if we suppose $\left|y_{n_{0}}^{2}-y_{n_{0}}^{1}\right|>\left|x_{n_{0}}^{2}-x_{n_{0}}^{1}\right|$, we obtain that $\left(x_{n_{0}}^{2}-x_{n_{0}}^{1}, y_{n_{0}}^{2}-y_{n_{0}}^{1}\right) \notin F$ implies $\left|y_{n}^{2}-y_{n}^{1}\right| \geqslant k(|b|-\varepsilon)^{n}$, for all $n \geqslant 0$. On the other hand, (2.a) implies $\left|y_{n}^{2}-y_{n}^{1}\right| \leqslant\left|y_{n}^{2}\right|+\left|y_{n}^{1}\right| \leqslant\left|x_{n}^{2}\right|+\left|x_{n}^{1}\right| \leqslant(|a|+\varepsilon)^{n}\left(\left|x_{0}^{2}\right|+\left|x_{0}^{1}\right|\right)$. From both inequalities we would obtain (the case $\left|x_{0}^{1}\right|+\left|x_{0}^{2}\right|=0$ being trivial), $((|b|-\varepsilon) /(|a|+\varepsilon))^{n} \leqslant$ $k /\left(\left|x_{0}^{1}\right|+\left|x_{0}^{2}\right|\right)$, for all $n \geqslant 0$, which is impossible because of $(|b|-\varepsilon) /(|a|+\varepsilon)>\hat{1}$. Thus, $\left|y_{n}^{2}-y_{n}^{1}\right| \leqslant\left|x_{n}^{2}-x_{n}^{1}\right|$, for all $n \geqslant 0$. Now, if $\left|v_{n}\right| \leqslant\left|u_{n}\right|$, for all $n \geqslant 0$, we have that $\left|u_{n+1}\right| \leqslant(|a|+\varepsilon)\left|u_{n}\right| \leqslant(|a|+\varepsilon)^{n}\left|u_{0}\right|$, and $\left|x_{n}^{2}-x_{n}^{1}\right| \leqslant(|a|+\varepsilon)^{n}\left|x_{0}^{2}-x_{0}^{1}\right|$. From both, (3.a) follows.

To show (3.b), given $\left(x_{n+1}, y_{n+1}\right)=T^{n}\left(x_{0}, y_{0}\right)$ such that $y_{0} \neq h_{u}\left(x_{0}\right)$, consider the orbit of $\left(x_{0}, h_{u}\left(x_{0}\right)\right)$, denote it by $\left(x_{n}^{1}, y_{n}^{1}\right)_{n}$ and construct the family $\left(\triangle T_{n}\right)_{n}$ as above. For the initial condition $\left(u_{0}, v_{0}\right)=\left(0, y_{0}-h_{u}\left(x_{0}\right)\right) \notin F$, we obtain $\left|v_{1}\right| \geqslant$ $(|b|-\varepsilon)\left|v_{0}\right|$, with $v_{1}=b\left(y_{0}-h_{u}\left(x_{0}\right)\right)+g_{0}\left(x_{0}, y_{0}\right)-g_{0}\left(x_{0}, h_{u}\left(x_{0}\right)\right)=y_{1}-h_{u}\left(x_{1}^{1}\right) ;$ hence $\left|y_{1}-h_{u}\left(x_{1}^{1}\right)\right| \geqslant(|b|-\varepsilon)\left|y_{0}-h_{u}\left(x_{0}\right)\right|$, with $x_{1}^{1}=a x_{0}+f_{0}\left(x_{0}, h_{u}\left(x_{0}\right)\right)$. On the other hand, $\left|y_{1}-h_{u}\left(x_{1}\right)\right| \geqslant\left|y_{1}-h_{u}\left(x_{1}^{1}\right)\right|-\left|h_{u}\left(x_{1}^{1}\right)-h_{u}\left(x_{1}\right)\right| \geqslant(|b|-\varepsilon)\left|y_{0}-h_{u}\left(x_{0}\right)\right|-$ $\left|x_{1}^{1}-x_{1}\right|$ and, since $x_{1}=a x_{0}+f_{0}\left(x_{0}, y_{0}\right),\left|x_{1}-x_{1}^{1}\right| \leqslant \varepsilon\left|y_{0}-h_{u}\left(x_{0}\right)\right|$. From both inequalities, $\left|y_{1}-h_{u}\left(x_{1}\right)\right| \geqslant(|b|-2 \varepsilon)\left|y_{0}-h_{u}\left(x_{0}\right)\right|>0$. By iterating this argument, we obtain the desired result.

\section{Dependence of the CURVE With REspect to THE Dyanmics}

An important aspect of this curve is its dependence with respect to the family of maps $\left(T_{n}\right)_{n}$. The next proposition shows an explicit relationship between differences in the families of maps and the corresponding curves. This result will also allow us to study the regularity of the curve in the case $|a|+\varepsilon \leqslant 1$.

PROPOSITION 4. Let $\left(T_{n}^{i}(x, y)=\left(a^{i}+f_{n}^{i}(x, y), b^{i} y+g_{n}^{i}(x, y)\right)\right)_{n \geqslant 0}, i=1,2$, be two families of maps satisfying the hypotheses of Proposition 2, with $\varepsilon>0$ satisfying (2.3) for both families at the same time. Given any $x_{0} \in \mathbb{R}$, we denote $y_{0}^{i}=h_{u}^{i}\left(x_{0}\right)$ the unique real number such that $T_{i}^{n}\left(x_{0}, y_{0}^{i}\right) \in F$, for every $n \geqslant 0$ and $i=1,2$. Then, if $\left\|D f_{n}^{2}(x, y)-D f_{n}^{1}(x, y)\right\| \leqslant \delta$, and $\left\|D g_{n}^{2}(x, y)-D g_{n}^{1}(x, y)\right\| \leqslant \delta$, for every $n \geqslant 0$ and every $(x, y) \in \mathbb{R}^{2}$, and if for instance $\left|a^{1}\right| \leqslant\left|a^{2}\right|$, we obtain

$$
\left|y_{0}^{2}-y_{0}^{1}\right|<\frac{2 \delta+\left|a^{2}-a^{1}\right|+\left|b^{2}-b^{1}\right|}{\left|b^{2}\right|-\left|a^{2}\right|-2 \varepsilon}\left|x_{0}\right|
$$

Proof: We shall show by induction that if

$$
\left|y_{0}^{2}-y_{0}^{1}\right| \geqslant \frac{2 \delta+\left|a^{2}-a^{1}\right|+\left|b^{2}-b^{1}\right|}{\left|b^{2}\right|-\left|a^{2}\right|-2 \varepsilon}\left|x_{0}\right|
$$


was true, then we would also have, for every $n \geqslant 0$,

$$
\begin{aligned}
& \left|y_{n}^{2}-y_{n}^{1}\right| \geqslant \frac{2 \delta+\left|a^{2}-a^{1}\right|+\left|b^{2}-b^{1}\right|}{\left|b^{2}\right|-\left|a^{2}\right|-2 \varepsilon}\left(\left|a^{1}\right|+\varepsilon\right)^{n}\left|x_{0}\right|, \\
& \left|x_{n}^{2}-x_{n}^{1}\right| \leqslant\left|y_{n}^{2}-y_{n}^{1}\right|
\end{aligned}
$$

where $\left(x_{n}^{i}, y_{n}^{i}\right)_{n}$ is the orbit under $\left(T_{n}^{i}\right)_{n}$ with initial condition $\left(x_{0}, y_{0}^{i}\right)$.

For $n=0,(4 . \mathrm{i})$ is true by hypothesis, and (4.ii) holds trivially. Suppose that both inequalities are true for a given $n$. Then,

$$
\begin{aligned}
\left|y_{n+1}^{2}-y_{n+1}^{1}\right|= & \left|b^{2} y_{n}^{2}+g_{n}^{2}\left(x_{n}^{2}, y_{n}^{2}\right)-b^{1} y_{n}^{1}-g_{n}^{1}\left(x_{n}^{1}, y_{n}^{1}\right)\right| \\
= & \mid b^{2}\left(y_{n}^{2}-y_{n}^{1}\right)+\left(b^{2}-b^{1}\right) y_{n}^{1}-\left(g_{n}^{2}\left(x_{n}^{2}, y_{n}^{2}\right)-g_{n}^{2}\left(x_{n}^{1}, y_{n}^{1}\right)\right) \\
& \quad+\left(g_{n}^{2}\left(x_{n}^{1}, y_{n}^{1}\right)-g_{n}^{1}\left(x_{n}^{1}, y_{n}^{1}\right)\right) \mid \\
\geqslant & \left|b^{2}\right|\left|y_{n}^{2}-y_{n}^{1}\right|-\left|b^{2}-b^{1}\right|\left|y_{n}^{1}\right|-\left\|D g_{n}^{2}\left(\xi_{1}, \xi_{2}\right)\right\|\left\|\left(x_{n}^{2}-x_{n}^{1}, y_{n}^{2}-y_{n}^{1}\right)\right\| \\
& \quad-\left\|\left(D g_{n}^{2}-D g_{n}^{1}\right)\left(\xi_{2}, \xi_{4}\right)\right\|\left\|\left(x_{n}^{1}, y_{n}^{1}\right)\right\| .
\end{aligned}
$$

Therefore, proceeding similarly for $x$,

and

$$
\begin{gathered}
\left|y_{n+1}^{2}-y_{n+1}^{1}\right| \geqslant\left|b^{2}\right|\left|y_{n}^{2}-y_{n}^{1}\right|-\varepsilon\left\|\left(x_{n}^{2}-x_{n}^{1}, y_{n}^{2}-y_{n}^{1}\right)\right\| \\
-\delta\left\|\left(x_{n}^{1}, y_{n}^{1}\right)\right\|-\left|b^{2}-b^{2}\right|\left|y_{n}^{1}\right|,
\end{gathered}
$$

$$
\begin{gathered}
\left|x_{n+1}^{2}-x_{n+1}^{1}\right| \leqslant\left|a^{2}\right|\left|x_{n}^{2}-x_{n}^{1}\right|+\varepsilon\left\|\left(x_{n}^{2}-x_{n}^{1}, y_{n}^{2}-y_{n}^{1}\right)\right\| \\
+\delta\left\|\left(x_{n}^{1}, y_{n}^{1}\right)\right\|+\left|a^{2}-a^{1}\right|\left|x_{n}^{1}\right| .
\end{gathered}
$$

Since $\left\|\left(x_{n}^{i}, y_{n}^{i}\right)\right\|=\left|x_{n}^{i}\right| \leqslant\left(\left|a^{i}\right|+\varepsilon\right)^{n}\left|x_{0}^{i}\right|$ and $\left\|\left(x_{n}^{2}-x_{n}^{1}, y_{n}^{2}-y_{n}^{1}\right)\right\|=\left|y_{n}^{2}-y_{n}^{1}\right|$ (this because of (4.ii) for $n$ ), we obtain

$$
\left|x_{n+1}^{2}-x_{n+1}^{1}\right| \leqslant\left(\left|a^{2}\right|+\varepsilon\right)\left|y_{n}^{2}-y_{n}^{1}\right|+\left(\delta+\left|a^{2}-a^{1}\right|\right)\left(\left|a^{1}\right|+\varepsilon\right)^{n}\left|x_{0}\right|,
$$

and

$$
\left|y_{n+1}^{2}-y_{n+1}^{1}\right| \geqslant\left(\left|b^{2}\right|-\varepsilon\right)\left|y_{n}^{2}-y_{n}^{1}\right|-\left(\delta+\left|b^{2}-b^{1}\right|\right)\left(\left|a^{1}\right|+\varepsilon\right)^{n}\left|x_{0}\right| .
$$

Thus, (4.ii) for $n+1$ is obtained from

$$
\begin{aligned}
& \left(\left|a^{2}\right|+\varepsilon\right)\left|y_{n}^{2}-y_{n}^{1}\right|+\left(\delta+\left|a^{2}-a^{1}\right|\right)\left(\left|a^{1}\right|+\varepsilon\right)^{n}\left|x_{0}\right| \\
& \quad \leqslant\left(\left|b^{2}\right|-\varepsilon\right)\left|y_{n}^{2}-y_{n}^{1}\right|-\left(\delta+\left|b^{2}-b^{1}\right|\right)\left(\left|a^{1}\right|+\varepsilon\right)^{n}\left|x_{0}\right|
\end{aligned}
$$

which is equivalent to $\left(2 \delta+\left|a^{2}-a^{1}\right|+\left|b^{2}-b^{1}\right|\right)\left(\left|a^{1}\right|+\varepsilon\right)^{n}\left|x_{0}\right|$ $\leqslant\left(\left|b^{2}\right|-\left|a^{2}\right|-2 \varepsilon\right)\left|y_{n}^{2}-y_{n}^{1}\right|$, and this, equivalent to (4.i) for $n$. 
Now,

$$
\begin{aligned}
\left|y_{n+1}^{2}-y_{n+1}^{1}\right| \geqslant & \left(\left|b^{2}\right|-\varepsilon\right)\left|y_{n}^{2}-y_{n}^{1}\right|-\left(\delta+\left|b^{2}-b^{1}\right|\right)\left(\left|a^{1}\right|+\varepsilon\right)^{n}\left|x_{0}\right| \\
\geqslant & \left(\left|b^{2}\right|-\varepsilon\right) \frac{2 \delta+\left|a^{2}-a^{2}\right|+\left|b^{2}-b^{1}\right|}{\left|b^{2}\right|-\left|a^{2}\right|-2 \varepsilon}\left(\left|a^{1}\right|+\varepsilon\right)^{n}\left|x_{0}\right| \\
& -\left(\delta+\left|b^{2}-b^{1}\right|\right)\left(\left|a^{1}\right|+\varepsilon\right)^{n}\left|x_{0}\right|,
\end{aligned}
$$

and property (4.i) for $n+1$ follows from

$$
\begin{gathered}
\left(\left|b^{2}\right|-\varepsilon\right) \frac{2 \delta+\left|a^{2}-a^{1}\right|+\left|b^{2}-b^{1}\right|}{\left|b^{2}\right|-\left|a^{2}\right|-2 \varepsilon}\left(\left|a^{1}\right|+\varepsilon\right)^{n}\left|x_{0}\right| \\
-\left(\delta+\left|b^{2}-b^{1}\right|\right)\left(\left|a^{1}\right|+\varepsilon\right)^{n}\left|x_{0}\right| \\
\geqslant \frac{2 \delta+\left|a^{2}-a^{1}\right|+\left|b^{2}-b^{1}\right|}{\left|b^{2}\right|-\left|a^{2}\right|-2 \varepsilon}\left(\left|a^{1}\right|+\varepsilon\right)^{n+1}\left|x_{0}\right|,
\end{gathered}
$$

which is equivalent to

$$
\begin{gathered}
\left(2 \delta+\left|a^{2}-a^{1}\right|+\left|b^{2}-b^{1}\right|\right)\left(\left|b^{2}\right|-\varepsilon\right)-\left(\delta+\left|b^{2}-b^{1}\right|\right)\left(\left|b^{2}\right|-\left|a^{2}\right|-2 \varepsilon\right) \\
\geqslant\left(2 \delta+\left|a^{2}-a^{1}\right|+\left|b^{2}-b^{1}\right|\right)\left(\left|b^{2}\right|-\left|a^{2}\right|-2 \varepsilon\right) \\
-\left(\delta+\left|b^{2}-b^{1}\right|\right)\left(\left|b^{2}\right|-\left|a^{2}\right|-2 \varepsilon\right) \geqslant 0
\end{gathered}
$$

and this is equivalent to $\left(\delta+\left|a^{2}-a^{1}\right|\right)\left(\left|b^{2}\right|-\left|a^{2}\right|-2 \varepsilon\right) \geqslant 0$, which is true by hypothesis (2.3).

Once we have proved that $\left|y_{0}^{2}-y_{0}^{1}\right| \geqslant\left(2 \delta+\left|a^{2}-a^{1}\right|+\left|b^{2}-b^{1}\right|\right) /\left(\left|b^{2}\right|-\left|a^{2}\right|-2 \varepsilon\right)$ implies (4.i) and (4.ii), notice that by iterating (4.iii) we obtain $\left|y_{n+1}^{2}-y_{n+1}^{1}\right| \geqslant\left(\left|b^{2}\right|-\varepsilon\right)^{n+1}\left|y_{0}^{2}-y_{0}^{1}\right|-\left(\delta+\left|a^{2}-a^{1}\right|\right)\left|x_{0}\right|\left(\left|b^{2}\right|-\varepsilon\right)^{n} \sum_{k=1}^{n}\left(\frac{\left|a^{1}\right|+\varepsilon}{\left|b^{2}\right|-\varepsilon}\right)^{k}$. The series $\sum_{k=1}^{\infty}\left(\left(\left|a^{1}\right|+\varepsilon\right) /\left(\left|b^{2}\right|-\varepsilon\right)\right)^{k}$ is a convergent geometric series of positive terms and its sum is $\left(\left|a^{1}\right|+\varepsilon\right) /\left(\left|b^{2}\right|-\left|a^{1}\right|-2 \varepsilon\right)$, hence

$$
\begin{aligned}
\left|y_{n+1}^{2}-y_{n+1}^{1}\right| & \geqslant\left(\left|b^{2}\right|-\varepsilon\right)^{n+1}\left(\left|y_{0}^{2}-y_{0}^{1}\right|-\frac{\left(\delta+\left|a^{2}-a^{1}\right|\right)\left|x_{0}\right|}{\left|b^{2}\right|-\varepsilon} \frac{\left|a^{1}\right|+\varepsilon}{\left|b^{2}\right|-\left|a^{1}\right|-2 \varepsilon}\right) \\
& \geqslant\left(\left|b^{2}\right|-\varepsilon\right)^{n+1}\left(\left|y_{0}^{2}-y_{0}^{1}\right|-\frac{\left(\delta+\left|a^{2}-a^{1}\right|\right)\left|x_{0}\right|}{\left|b^{2}\right|-\left|a^{2}\right|-2 \varepsilon}\right) \geqslant 0 .
\end{aligned}
$$

(This is because of (4.i) for $n=0$ and because $\left|b^{2}\right|-\left|a^{1}\right|-2 \varepsilon>\left|b^{2}\right|-\left|a^{2}\right|-2 \varepsilon$.) 
On the other hand,

$$
\begin{aligned}
\left|y_{n}^{2}-y_{n}^{1}\right| & \leqslant\left|y_{n}^{2}\right|+\left|y_{n}^{1}\right| \leqslant\left|x_{n}^{2}\right|+\left|x_{n}^{1}\right| \\
& \leqslant\left|x_{0}\right|\left(\left(\left|a^{1}\right|+\varepsilon\right)^{n}+\left(\left|a^{2}\right|+\varepsilon\right)^{n}\right) \\
& \leqslant 2\left|x_{0}\right|\left(\left|a^{2}\right|+\varepsilon\right)^{n} .
\end{aligned}
$$

From both inequalities we obtain,

$$
\left(\frac{\left|b^{2}\right|-\varepsilon}{\left|a^{1}\right|+\varepsilon}\right)^{n} \leqslant \frac{2\left|x_{0}\right|}{\left|y_{0}^{2}-y_{0}^{1}\right|-\left(\delta+\left|a^{2}-a^{1}\right|\right)\left|x_{0}\right|\left(\left|b^{2}\right|-\left|a^{2}\right|-2 \varepsilon\right)^{-1}}
$$

for all $n \geqslant 0$, which is not possible. This ends the proof.

REMARK. Since the solution for the linear case $T_{n}(x, y)=(a x, b y)$ is the line $y=0$, we obtain, in particular, that $\left|h_{u}(x)\right| \leqslant O(\varepsilon)|x|$, where $O(\varepsilon)$ goes to zero as $\varepsilon$ goes to zero.

\section{REGULARITY OF THE INVARIANT REPELLING CURVE}

Proposition 5. Let $\left(T_{n}(x, y)=\left(a x+f_{n}(x, y), b y+g_{n}(x, y)\right)\right)_{n}, n \geqslant 0$ be a family of $C^{1}$ maps of the plane satisfying the hypotheses of Proposition 2. If, additionally, $|a|+\varepsilon \leqslant 1$ and $\left(D T_{n}\right)_{n}$ is equicontinuous at every point, then $h_{u}$ defined as above is of class $C^{1}$ at every point.

Proof: Given any $x_{0}^{1} \in \mathbb{R}$ and given $x_{0}^{2} \neq x_{0}^{1}$ any point close to $x_{0}^{1}$, consider the sequences of points given by $\left(x_{n}^{i}, y_{n}^{i}\right)=T^{n}\left(x_{0}^{i}, y_{0}^{i}\right)$ and $y_{0}^{i}=h_{u}\left(x_{0}^{i}\right), i=1,2$. Let us construct the following families of maps,

$$
\begin{aligned}
& S_{n}^{1}(u, v)=\left(a u+D_{1} f_{n}\left(x_{n}^{1}, y_{n}^{1}\right) u+D_{2} f_{n}\left(x_{n}^{1}, y_{n}^{1}\right) v, b v+D_{1} g_{n}\left(x_{n}^{1}, y_{n}^{1}\right) u\right. \\
&\left.+D_{2} g_{n}\left(x_{n}^{1}, y_{n}^{1}\right) v\right) \\
& S_{n}^{2}(u, v)=\left(a u+D_{1} f_{n}\left(\xi_{1}(n), y_{n}^{1}\right) u+D_{2} f_{n}\left(x_{n}^{1}, \xi_{2}(n)\right) v\right. \\
&\left.b v+D_{1} g_{n}\left(\xi_{3}(n), y_{n}^{1}\right) u+D_{2} g_{n}\left(x_{n}^{1}, \xi_{4}(n)\right) v\right)
\end{aligned}
$$

where $\xi_{j}(n), j=1,2,3,4$, are real numbers satisfying for every $n \geqslant 0$,

$$
\begin{aligned}
& \left|\xi_{j}(n)-x_{n}^{1}\right| \leqslant\left|x_{n}^{2}-x_{n}^{1}\right|, \quad j=1,3, \\
& \left|\xi_{j}(n)-y_{n}^{1}\right| \leqslant\left|y_{n}^{2}-y_{n}^{1}\right|\left(\leqslant\left|x_{n}^{2}-x_{n}^{1}\right|\right), \quad j=2,4, \\
& f_{n}\left(x_{n}^{2}, y_{n}^{2}\right)-f_{n}\left(x_{n}^{1}, y_{n}^{1}\right)=D_{1} f_{n}\left(\xi_{1}(n), y_{n}^{1}\right)\left(x_{n}^{2}-x_{n}^{1}\right) \\
& \quad+D_{2} f_{n}\left(x_{n}^{2}, \xi_{2}(n)\right)\left(y_{n}^{2}-y_{n}^{1}\right), \\
& \quad g_{n}\left(x_{n}^{2}, y_{n}^{2}\right)-g_{n}\left(x_{n}^{1}, y_{n}^{1}\right)=D_{1} g_{n}\left(\xi_{3}(n), y_{n}^{1}\right)\left(x_{n}^{2}-x_{n}^{1}\right) \\
& \quad+D_{2} g_{n}\left(x_{n}^{2}, \xi_{4}(n)\right)\left(y_{n}^{2}-y_{n}^{1}\right) .
\end{aligned}
$$


$\left(S_{n}^{i}\right)_{n}, i=1,2$, are two families of linear mappings that, since $|a|+\varepsilon \leqslant 1$, are close to each other, if $x_{0}^{2}$ is close enough to $x_{0}^{1}$. More precisely, if we set $\delta\left(x_{n}^{1}, x_{n}^{2}\right)$ the supremum of the quantities

$$
\begin{aligned}
& \left|D_{1} f_{n}\left(x, y_{n}^{1}\right)-D_{1} f_{n}\left(x_{n}^{1}, y_{n}^{1}\right)\right|,\left|D_{2} f_{n}\left(x_{n}^{2}, y\right)-D_{2} f_{n}\left(x_{n}^{1}, y_{n}^{1}\right)\right| \\
& \left|D_{1} g_{n}\left(x, y_{n}^{1}\right)-D_{1} g_{n}\left(x_{n}^{1}, y_{n}^{1}\right)\right| \text { and }\left|D_{2} g_{n}\left(x_{n}^{2}, y\right)-D_{2} g_{n}\left(x_{n}^{1}, y_{n}^{1}\right)\right|
\end{aligned}
$$

for every $n \geqslant 0,\left|x-x_{n}^{1}\right| \leqslant\left|x_{n}^{2}-x_{n}^{1}\right|$ and $\left|y-y_{n}^{1}\right| \leqslant\left|y_{n}^{2}-y_{n}^{1}\right|$, then, because of the uniform equicontinuity of $\left(D T_{n}\right)_{n}$ in the compact set $\overline{B\left(0,\left|x_{0}^{1}\right|+\left|x_{0}^{2}\right|\right)}$, and because of $\left|y_{n}^{2}-y_{n}^{1}\right| \leqslant\left|x_{n}^{2}-x_{n}^{1}\right| \leqslant(|a|+\varepsilon)^{n}\left|x_{0}^{2}-x_{0}^{1}\right|,\left|y_{n}^{i}\right| \leqslant\left|x_{n}^{i}\right| \leqslant(|a|+\varepsilon)^{n}\left|x_{0}^{i}\right| \leqslant\left|x_{0}^{i}\right| \leqslant$ $\left|x_{0}^{1}\right|+\left|x_{0}^{2}\right|$, this supremum is finite and $\lim _{x_{n}^{2} \rightarrow x_{n}^{1}} \delta\left(x_{n}^{1}, x_{n}^{2}\right)=0$.

Thus, the conditions of the previous proposition are satisfied. Take $u_{0}=1$, for $\left(S_{n}^{2}\right)_{n}$ the sequence $\left(\left(x_{n}^{2}-x_{n}^{1}\right) /\left(x_{0}^{2}-x_{0}^{1}\right),\left(y_{n}^{2}-y_{n}^{1}\right) /\left(x_{0}^{2}-x_{0}^{1}\right)\right)_{n}$ is the unique invariant sequence $\left(u_{n}, v_{n}\right)$ satisfying $\left|v_{n}\right| \leqslant\left|u_{n}\right|$. Let $v_{0}^{1}$ be the corresponding initial condition for $\left(S_{n}^{1}\right)_{n}$, then, according to Proposition 4,

$$
\left|\frac{y_{0}^{2}-y_{0}^{1}}{x_{0}^{2}-x_{0}^{1}}-v_{0}^{1}\right|<\frac{2 \delta\left(x_{0}^{1}, x_{0}^{2}\right)}{|b|-|a|-2 \varepsilon}\left|u_{0}\right| \text {. }
$$

Then, the limit $\lim _{x_{0}^{2} \rightarrow x_{0}^{1}}\left(y_{0}^{2}-y_{0}^{1}\right) /\left(x_{0}^{2}-x_{0}^{1}\right)$ exists and it equals $v_{0}^{1}$. Hence $h_{u}$ has a derivative at $x_{0}^{1}$.

Set now, for all $n \geqslant 0, S_{n}^{3}(u, v)=\left(a u+D_{1} f_{n}\left(x_{n}^{2}, y_{n}^{1}\right) u+D_{2} f_{n}\left(x_{n}^{2}, y_{n}^{2}\right) v, b v\right.$ $\left.+D_{1} g_{n}\left(x_{n}^{2}, y_{n}^{2}\right) u+D_{2} g_{n}\left(x_{n}^{2}, y_{n}^{2}\right) v\right) .\left(S_{n}^{1}\right)_{n}$ and $\left(S_{n}^{3}\right)_{n}$ are two families of linear mappings satisfying the conditions of Proposition 4, with $\delta$ tending to zero if $x_{0}^{2}$ tends to $x_{0}^{1}$. In this situation, the initial condition $\left(1, v_{0}^{1}\right)$ and $\left(1, v_{0}^{3}\right)$ satisfy $\left|v_{0}^{3}-v_{0}^{1}\right|<$ $(2 \delta) /(|b|-|a|-2 \varepsilon)$, but we have just proved that $v_{0}^{1}=h_{u}^{\prime}\left(x_{0}^{1}\right)$ and $v_{0}^{3}=h_{u}^{\prime}\left(x_{0}^{2}\right)$. Thus, we obtain the continuity of $h_{u}^{\prime}$.

\section{Application to the general CaSe}

In order to apply the results obtained, conditions (2.1) to (2.3) must be satisfied. The first condition is natural, and the last one can be obtained locally in an easy way. We shall see now how (2.2) can also be obtained. We state and prove here our results for the general case.

THEOREM 6. Let $T: U \subseteq \mathbb{R}^{2} \rightarrow U$ be a $C^{1}$ map with a fixed point $p \in \operatorname{Int}(U)$. Let $a$ and $b$ be the eigenvalues, both real, of $D T(p)$, and let them satisfy $|a|<|b|$.

EXISTENCE. The map $T$ has a locally invariant continuous curve coming through the point $p$ that can be described as the graph $y=h_{u}(x)$ of a Lipschitz function with 
constant smaller than or equal to one. For this purpose, an affine change of co-ordinates, $\alpha_{T}(x, y)$, has to be applied. This change of co-ordinates associates $p$ to the origin and the principal directions of $a$ and $b$ to the $O X$ and $O Y$ axis, respectively. This curve is locally repelling in the sense of (3.b).

DIFFERENTIABILITY. If, additionally, $|a|<1$, then there exists a curve satisfying the conditions described that is of class $C^{1}$ and tangent at $p$ to the principal direction associated to $a$, that is, $h_{u}$ has a derivative, this derivative is continuous, and $h_{u}^{\prime}(0)=$ 0 .

DEPENDENCE WITH RESPECT TO $T$. The curve depends continuously on $p$ and $D T$ in the following terms,

(6.a) the affine change of co-ordinates can be written in the form $\alpha_{T}(x, y)=$ $L_{T}((x, y)-p)$, where $L_{T}$ is a linear mapping depending continuously on $D T(p)$.

(6.b) given any other $C^{1}$ map, $S: U \subseteq \mathbb{R}^{2} \rightarrow U$, with a fixed point $p^{\prime} \in \operatorname{Int}(U)$ satisfying the existence conditions, if there exist $\beta>0$ and $K \subseteq U, K$ a compact set containing both $p$ and $p^{\prime}$ in its interior, such that $\left|p-p^{\prime}\right| \leqslant \beta$ and $\|D T(x, y)-D S(x, y)\| \leqslant \beta$, for every $(x, y) \in K$, then for every bounded $I \subset R$,

$$
\lim _{\beta \rightarrow 0}\left\{\sup \left|h_{u_{T}}(x)-h_{u_{S}}(x)\right|: x \in I\right\}=0 .
$$

\section{ProOF.}

EXISTENCE. using an affine change of co-ordinates like the one described and that depends continuously on $p$ and $D T(p)$, we can make our fixed point be the origin and the matrix associated to the differential of the resulting map at it be $\operatorname{diag}\langle a, b\rangle$. Denote the new map $T_{0}=\alpha_{T} \circ T \circ \alpha_{T}^{-1}$, and define $(f(x, y), g(x, y))=T_{0}(x, y)-(a x, b y) . f$ and $g$ are two $C^{1}$ maps defined on a neighbourhood of the origin satisfying $f(0,0)=$ $g(0,0)=0$ and $D f(0,0)=D g(0,0)=0\left(\in L\left(\mathbb{R}^{2}, \mathbb{R}\right)\right)$.

Let $r: \mathbb{R} \rightarrow[0,1]$ be a $C^{\infty}$ function such that $r(x)=0$, for every $|x| \geqslant 1$ (hence, $r^{\prime}(x)=0$, for $\left.|x| \geqslant 1\right)$ and $r(x)=1$, for every $|x| \leqslant 1 / 2$, and set $k=\sup \left\{\left|r^{\prime}(x)\right|: x \in\right.$ $\mathbb{R}\}<+\infty$.

Since $D f$ and $D g$ are continuous at $(0,0)$, given any $\varepsilon^{\prime}>0$, there exists $\delta\left(\varepsilon^{\prime}\right)>0$ such that $\|(x, y)\| \leqslant \delta$ implies $\|D f(x, y)\| \leqslant \varepsilon^{\prime}$ and $\|D g(x, y)\| \leqslant \varepsilon^{\prime}$. In particular, $\|(x, y)\| \leqslant \delta$ implies $\|f(x, y)\|=\|f(x, y)-f(0,0)\| \leqslant\left|D f\left(\xi_{1}, \xi_{2}\right)\right|\|(x, y)\| \leqslant$ $\varepsilon^{\prime}\|(x, y)\|$, for some $\left\|\left(\xi_{1}, \xi_{2}\right)\right\| \leqslant\|(x, y)\|$, and $\|g(x, y)\| \leqslant \varepsilon^{\prime}\|(x, y)\|$. 
Consider the new map, also of class $C^{1}$, defined all through $\mathbb{R}^{2}$ by

$$
\begin{aligned}
\widehat{T}(x, y) & =(a x+\widehat{f}(x, y), b y+\widehat{g}(x, y)) \\
& =\left(a x+r\left(\frac{x^{2}+y^{2}}{\delta^{2}}\right) f(x, y), b y+r\left(\frac{x^{2}+y^{2}}{\delta^{2}}\right) g(x, y)\right) .
\end{aligned}
$$

This new map coincides locally with $T_{0}$. Our goal is to find a curve invariant for $\widehat{T}$, which will be locally invariant for $T_{0}$.

Under the conditions described,

$$
D_{1} \widehat{f}(x, y)=r^{\prime}\left(\frac{x^{2}+y^{2}}{\delta^{2}}\right) \frac{2 x}{\delta^{2}} f(x, y)+r\left(\frac{x^{2}+y^{2}}{\delta^{2}}\right) D_{1} f(x, y)
$$

and we obtain,

$$
\begin{aligned}
& \text { if }\|(x, y)\|>\delta, \text { then } x^{2}+y^{2}>\delta^{2} \text { and } D_{1} \widehat{f}(x, y)=0 \\
& \text { if }\|(x, y)\| \leqslant \delta \text {, then }\left|D_{1} \widehat{f}(x, y)\right| \leqslant 2 k \frac{|x|}{\delta} \varepsilon^{\prime} \frac{\|(x, y)\|}{\delta}+\varepsilon^{\prime} \leqslant \varepsilon^{\prime}(1+2 k) .
\end{aligned}
$$

Thus, $\left|D_{1} \widehat{f}(x, y)\right| \leqslant \varepsilon^{\prime}(1+2 k)$, for every $(x, y) \in \mathbb{R}^{2}$, and similarly for $D_{2} \widehat{f}(x, y)$, $D_{1} \widehat{g}(x, y)$ and $D_{2} \widehat{g}(x, y)$. Given $0<\varepsilon<(|b|-|a|) / 2$, consider $\varepsilon^{\prime}=\varepsilon /(1+2 k)>0$. Taking $\widehat{T}$ as in (6.i), with $\delta=\delta\left(\varepsilon^{\prime}\right)$, then $\|D \widehat{f}(x, y)\| \leqslant \varepsilon$ and $\|D \widehat{g}(x, y)\| \leqslant \varepsilon$, for every $(x, y) \in \mathbb{R}^{2}$, and $\widehat{f}(x, y)=\widehat{g}(x, y)=0$, for every $\|(x, y)\|>\delta$.

Finally, the fact that $\hat{f}$ and $\widehat{g}$ are zero for $\|(x, y)\| \geqslant \delta$ gives (2.2). To see this, for fixed $n$ and $x_{0}$, the mapping $\alpha: \mathbb{R} \longrightarrow \mathbb{R}$ with $\alpha(y)=\Pi_{2} \circ \widehat{T}^{n-1}\left(x_{0}, y\right)$ (where $\Pi_{2}$ denotes the projection on the second coordinate) is continuous and, if $|y|$ is large enough, linear, since if $|y| \geqslant K=\max \left\{|b|^{-n}, 1\right\} \delta$, then

$$
\begin{aligned}
\widehat{T}\left(x_{0}, y\right) & =\left(a x_{0}+\widehat{f}\left(x_{0}, y\right), b y+\widehat{g}\left(x_{0}, y\right)\right)=\left(a x_{0}, y\right), \\
\widehat{T}^{1}\left(x_{0}, y\right) & =\widehat{T}\left(a x_{0}, b y\right)=\left(a^{2} x_{0}+\widehat{f}\left(a x_{0}, b y\right), b y^{2}+\widehat{g}\left(a x_{0}, b y\right)\right) \\
& =\left(a^{2} x_{0}, b^{2} y\right), \\
\cdots & \\
\widehat{T}^{n-1}\left(x_{0}, y\right) & =\left(a^{n} x_{0}, b^{n} y\right),
\end{aligned}
$$

due to $\left|b^{j} y\right| \geqslant \delta$, for every $1 \leqslant j \leqslant n$. Thus, $|y| \geqslant K$ implies $\alpha(y)=b^{n} y$.

From this we conclude that $\alpha(K) \alpha(-K)=-b^{2 n} K^{2} \leqslant 0$ and, hence, there exists $c \in[-K, K]$ such that $\alpha(c)=0$.

Therefore, the family $\left(T_{n}\right)_{n}=(\widehat{T})_{n}$ satisfies the hypotheses of Propositions 3 and 4. This gives the existence of $h_{u}$. 
Differentiability. Also, if $|a|<1$ we can choose $\varepsilon>0$ such that $|a|+\varepsilon \leqslant 1$ and, since $T_{n}=\widehat{T}$ for all $n \geqslant 0$, the hypotheses of Proposition 5 hold, which yields the differentiability of $h_{u}$ and the continuity of $h_{u}^{\prime}$. Moreover, since $h_{u}$ satisfies $h_{u}\left(a x+\widehat{f}\left(x, h_{u}(x)\right)\right)=b h_{u}(x)+\widehat{g}\left(x, h_{u}(x)\right)$, then

$$
\begin{gathered}
h_{u}^{\prime}\left(a x+\widehat{f}\left(x, h_{u}(x)\right)\right)\left(a+D_{1} \widehat{f}\left(x, h_{u}(x)\right)\right)+D_{2} \widehat{f}\left(x, h_{u}(x)\right) h_{u}^{\prime}(x) \\
=b h_{u}(x)+D_{1} \widehat{g}\left(x, h_{u}(x)\right)+D_{2} \widehat{g}\left(x, h_{u}(x)\right) h_{u}^{\prime}(x) .
\end{gathered}
$$

Thus, for $x=0,(a-b) h_{u}^{\prime}(0)=0$, hence $h_{u}^{\prime}(0)=0$.

DePENDENCE WITH RespeCt to $T$. The proof of (6.a) is straightforward. Take now $S$ satisfying the described conditions; consider $T^{1}=\widehat{T}$ as above and set $T^{2}=\widehat{S}$, where $S_{0}$ and $\widehat{S}$ are constructed similarly to $T_{0}$ and $\widehat{T}$. Thanks to Proposition 4 we only need to show that there is an upper bound $M=M(\beta)$ for the quatities $\left\|D f^{2}(x, y)-D f^{1}(x, y)\right\|,\left\|D g^{2}(x, y)-D g^{1}(x, y)\right\|,\left|a^{1}-a^{1}\right|$ and $\left|b^{2}-b^{1}\right|$ (at least for $\|(x, y)\| \leqslant \gamma, \gamma$ a small enough positive number such that $B((0,0), \gamma) \subset \alpha_{T}(K) \cap$ $\alpha_{S}(K)$ ), and that

$$
\lim _{\beta \rightarrow 0} M(\beta)=0 .
$$

Denoting $L(a, b)(x, y)=(a x, b y), L_{T}=L(a, b)$ and $L_{S}=L\left(a^{\prime}, b^{\prime}\right)$ we have that, $\max \left\{\left|a^{2}-a^{1}\right|,\left|b^{2}-b^{1}\right|\right\} \leqslant\left\|D S\left(p^{\prime}\right)-D T(p)\right\| \leqslant\left\|D S\left(p^{\prime}\right)-D T\left(p^{\prime}\right)\right\|+\| D T\left(p^{\prime}\right)$ $-D T(p) \|$ and, if $\|(x, y)\| \leqslant \gamma$,

$$
\begin{aligned}
& \left\|\left(D f^{2}(x, y), D g^{2}(x, y)\right)-\left(D f^{1}(x, y), D g^{1}(x, y)\right)\right\| \\
& \quad=\left\|D S_{0}(x, y)-L_{S}-D T_{0}(x, y)+L_{T}\right\| \leqslant\left\|D S_{0}(x, y)-D T_{0}(x, y)\right\|+\left\|L_{S}-L_{T}\right\|
\end{aligned}
$$

and

$$
\begin{aligned}
& \left\|D S_{0}(x, y)-D T_{0}(x, y)\right\|=\mid L_{T} \circ D T\left(\alpha_{T}^{-1}(x, y)\right) \circ L_{T}^{-1}-L_{S} \circ D S\left(\alpha_{S}^{-1}(x, y)\right) \circ L_{S}^{-1} \| \\
& \leqslant\left\|L_{T}-L_{S}\right\|\left\|D T\left(\alpha_{T}^{-1}(x, y)\right)\right\|\left\|L_{T}^{-1}\right\| \\
& \quad+\left\|L_{S}\right\|\left\|D T\left(\alpha_{T}^{-1}(x, y)\right)-D T\left(\alpha_{S}^{-1}(x, y)\right)\right\|\left\|L_{T}^{-1}\right\| \\
& \quad+\left\|L_{S}\right\|\left\|D T\left(\alpha_{S}^{-1}(x, y)\right)\right\|\left\|L_{T}^{-1}-L_{S}^{-1}\right\| \\
& \quad+\left\|L_{S}\right\|\left\|D T\left(\alpha_{S}^{-1}(x, y)\right)-D S\left(\alpha_{S}^{-1}(x, y)\right)\right\|\left\|L_{S}^{-1}\right\| .
\end{aligned}
$$

Using the uniform continuity of $D T$ in $K$, the continuity of the norm, (6.a) and the hypotheses of (6.b), we obtain the desired upper bound. 


\section{REFERENCES}

[1] D.G. Aronson, M.A. Chory, G.R. Hall and R.P. McGehee, 'Bifurcations from an invariant circle for two-parameter families of maps of the plane: A computer-assisted study', Comm. Math. Phys. 83 (1982), 303-354.

[2] F. Esquembre, Rotation sets for circle maps and invariant curves in planar transformations, Ph.D. Thesis (Universidad de Murcia, 1991).

[3] P. Hartmann, Ordinary differential equations, 2nd edition (Birkhäuser, 1982).

[4] M.W. Hirsch, C.C. Pugh and M. Shub, Invariant manifolds, Lectures Notes in Mathematics 583 (Springer-Verlag, Berlin, 1977).

[5] G. Iooss, Bifurcation of maps and applications (North Holland, 1979).

\footnotetext{
Department of Mathematics

University of Murcia

30071 Murcia

Spain
} 